\title{
Conducta en pacientes con parálisis y patología del nervio radial
}

\author{
Sebastián Valbuena, ${ }^{*}$ Mariano Socolovsky** \\ "Hospital El Cruce Alta Complejidad en Red “Dr. Néstor Carlos Kirchner”, Florencio Varela, Buenos Aires, Argentina \\ Clínica del Deporte, La Plata, Buenos Aires, Argentina \\ Sanatorio de la Trinidad de Quilmes, Buenos Aires, Argentina \\ ** Servicio de Neurocirugía, Hospital de Clínicas “José de San Martín”, Universidad de Buenos Aires, Ciudad Autónoma de Buenos Aires, \\ Argentina
}

\section{INTRODUCCIÓN}

La patología traumática del nervio periférico es una situación compleja, e involucra un espectro posible de lesiones en el nervio afectado que abarcan desde un bloqueo transitorio en la conducción hasta la sección completa del nervio. Según Shao y cols. ${ }^{1}$, en el 11,8\% de las fracturas de húmero está afectado el nervio radial, y representa la lesión nerviosa que, con más frecuencia, se asocia a la fractura de huesos largos.

El manejo conservador y el seguimiento de la lesión del nervio radial, como el tiempo y la indicación correctos del tratamiento quirúrgico con su abanico de opciones reconstructivas plantean un escenario dinámico, muchas veces, dificultoso para el médico tratante. El contrapunto expone dos perspectivas para la resolución de un problema en común; en este caso, se exponen distintas situaciones dentro de la práctica médica respecto de la patología traumática del nervio radial.

\section{La parálisis del nervio radial luego de una fractura diafisaria cerrada de húmero es un cuadro frecuente en la práctica laboral. ¿Cuál es su manejo habitual en estos casos?}

Mariano Socolovsky (MS): Si bien se trata de la parálisis (y anestesia asociada) más frecuente luego de una fractura de hueso largo, su incidencia, según la bibliografía, no excede el $12 \% .{ }^{1}$ Nuestra línea de tratamiento es, en general, conservadora; la tasa de recuperación espontánea reportada es alta, oscila entre el $72 \%$ y el $93 \%{ }^{1,2}$ Consideramos fundamental el rol de las imágenes (ecografía y neurografía por resonancia magnética) en el diagnóstico y el tratamiento de estas lesiones, permiten determinar la continuidad (o no) del nervio, así como el grado de lesión. Si observáramos una solución de continuidad del nervio radial, un atrapamiento o alguna otra lesión, dejaríamos de lado el tratamiento conservador inicial y ofreceríamos una exploración quirúrgica del nervio con eventual reconstrucción, de acuerdo con los hallazgos intraoperatorios. Otra excepción sería cuando el tratamiento de la propia fractura amerite una exploración a cielo abierto, reducción y osteosíntesis, circunstancia que deberá ser aprovechada para inspeccionar el nervio. Finalmente, aclarar que, si bien nuestra propuesta terapéutica es, en general, conservadora, ante el tratamiento quirúrgico de la fractura o la confirmación de sección neural en estudios por imágenes, indicamos la exploración del nervio.

Sebastián Valbuena (SV): El 70\% de las parálisis del nervio radial asociadas a una fractura humeral se recupera espontáneamente, por lo que suele recomendarse la observación. ${ }^{1,3-6} \operatorname{Sin}$ embargo, hay evidencia de que, en las exploraciones tempranas, se observa una recuperación en el 89,8\% comparadas con el 68,1\% de las tardías. ${ }^{4}$

Como actualmente la mayoría de las fracturas de húmero suelen ser quirúrgicas, mi conducta ante una parálisis del nervio radial depende más de las características de la fractura que de la lesión neural.

En las fracturas tipo A, B1 y B2, prefiero explorar el nervio radial evaluando la lesión, evitando el atrapamiento óseo y el potencial daño de las manipulaciones cerradas, y uso sistemas DCP o LC-DCP bloqueados de 4,5 mm. En las fracturas de alta energía, conminutas, multifragmentarias (B3 y C), la reconstrucción neural logra resultados pobres. ${ }^{7,8}$ En consecuencia, uso sistemas MIPO o endomedulares sin explorar el nervio radial (excepto en fracturas abiertas), difiriendo el tratamiento de la parálisis.

Recibido el 7-8-2021. Aceptado luego de la evaluación el 7-8-2021 • Dr. SEBASTIÁN VALBUENA • valbuena.sebastian@ gmail.com Cómo citar este artículo: Valbuena S, Socolovsky M. Conducta en pacientes con parálisis y patología del nervio radial. Rev Asoc Argent Ortop Traumatol 2021;86(5):705-710. https://doi.org/10.15417/issn. 1852-7434.2021.86.5.1417 
En niños con fracturas abiertas y aquellas que serán operadas, sistemáticamente exploro el nervio. En ancianos con fracturas osteoporóticas e indicación de tratamiento conservador, una parálisis del nervio radial no cambia la conducta y realizo un control clínico.

En casos de parálisis del plexo braquial y fractura humeral, siempre exploro el nervio radial y realizo una reducción abierta y fijación interna para descartar una doble lesión.

\section{En caso de optar por el manejo conservador como elección primaria: ¿cómo realiza el seguimiento clínico del paciente y cuáles considera como puntos clave para valorar su recuperación?}

MS: La clínica, en primer lugar, y la neurofisiología, en segundo, son los puntales del seguimiento en esta etapa. La evaluación sensitiva nos podrá dar una presunción inicial de recuperación, aunque no garantiza una mejoría de la parálisis. Debemos calcular desde el punto de la presunta lesión nerviosa hasta la musculatura efectora, el tiempo que requerirá observar movimiento en los músculos paralizados, teniendo en cuenta que la velocidad de crecimiento axonal ronda el milímetro diario y puede acelerarse hasta a $3 \mathrm{~mm} /$ día en los niños. La rehabilitación es fundamental en este período, siempre que el tratamiento de la fractura lo permita. La ventaja de la neurofisiología es que nos permite observar la recuperación motora antes de que se detecte en el examen físico, si bien este hecho tampoco garantiza que la recuperación sea completa o útil. Si no se observa una mejoría clínica al cabo de los tiempos calculados, en el contexto mencionado en la pregunta anterior, si hay un estudio por imágenes que no muestre una lesión grave del nervio, deberemos abandonar la conducta conservadora y ofrecer una exploración nerviosa. En resumen, conducta conservadora no debe ser asimilable a conducta pasiva. La espera debe ser activa.

SV: Fundamentalmente dos pilares:

1) Férula dinámica, con muñeca en extensión y metacarpofalángicas libres, asociada a ejercicios de movilidad articular pasiva.

2) Evaluación: la alteración parcial de la sensibilidad en la cara dorsal de la primera comisura, la sudoración y la disfunción muscular, pero sin atrofia, indican una neuropraxia. La ausencia de sensibilidad, la parálisis motora y el signo de Tinel sugieren una axonotmesis o neurotmesis, difícil de diferenciar una de la otra. Un signo de Tinel que va progresando señala recuperación.

La ecografía puede ayudar a valorar la continuidad del nervio, un neuroma o una neurotmesis.

Los primeros músculos en recuperar la función son el braquiorradial y el extensor radial del carpo largo, y el último es el extensor propio del índice; el seguimiento clínico se basa en evaluarlos. El tiempo esperado de recuperación puede estimarse midiendo la distancia entre la fractura y el punto de inervación del braquiorradial, a $2 \mathrm{~cm}$ por encima del epicóndilo, calculando una velocidad de $1 \mathrm{~mm} /$ día.

El tiempo promedio de comienzo de la recuperación es de 7.3 semanas. ${ }^{1}$ Se observan diferencias entre lesiones de baja energía (3.1 semanas) y de alta (12 semanas). ${ }^{8}$ La recuperación completa suele observarse entre los 6 y 12 meses. ${ }^{1,6}$

\section{3. ¿Qué tiempo considera apropiado para realizar una electromiografía y qué valor pronóstico le asigna al resultado?}

MS: La electromiografía nunca se debe realizar antes de los 21 días posteriores a la lesión nerviosa, tiempo mínimo para que el estudio detecte signos claros de desnervación. La segunda parte de la pregunta entiendo que la respondí en el punto anterior.

SV: La electromiografía con velocidad de conducción, en mi experiencia, solo aporta un valor medicolegal. En caso de solicitarla, debe hacerse a las 3 o 4 semanas, ya que, a partir de ese momento, se observan potenciales de fibrilación muscular, ondas positivas, y potenciales de acción monofásicos de corta duración. ${ }^{3,9}$

Algunos autores proponen su uso para definir la conducta quirúrgica cuando no hay cambios entre una electromiografía basal y otra de control a las 6 semanas. $^{3}$

Los estudios de conducción nerviosa no permiten diferenciar tempranamente entre un nervio muy lesionado y uno que aún no se ha recuperado, ni pronostica el potencial de recuperación de un nervio, solo se anticipa unas semanas a la recuperación clínica; ${ }^{7}$ por lo tanto, no suelo usarla para tomar decisiones. Solo solicito una electromiografía por motivos medicolegales (pacientes laborales, accidentes de tránsito, etc.) a las 4 semanas y 8 semanas después si no hay recuperación. 


\section{4. ¿Cuál es el tiempo que considera límite para mantener el tratamiento conservador o qué parámetros negativos considera de relevancia para suspenderlo?}

MS: En sincronía con lo ya mencionado, y salvando todas las condiciones clínicas, de imágenes y neurofisiológicas antes descritas, diría que 6 meses desde que se produce la lesión es un tiempo al cabo del cual se debería abandonar la conducta conservadora. ${ }^{10}$ Esto es claramente una regla general, el cirujano debe evaluar cada caso en particular, porque habrá ocasiones en las que se deba indicar cirugía antes de ese tiempo o inclusive más tarde si hubiere signos, al menos, incipientes de recuperación.

SV: No existe consenso bibliográfico sobre el tiempo que se debe sostener un tratamiento conservador. Tradicionalmente se ha propuesto una conducta expectante entre los 3 y los 6 meses, seguida de una exploración neural tardía cuando no hay recuperación funcional. ${ }^{3}$ Sin embargo, también se ha comunicado que si, en 2 o 3 meses, no hay indicios de recuperación motora, en la gran mayoría de los casos, no los habrá. ${ }^{11,12}$ Personalmente considero que, en pacientes con fracturas de baja energía, la espera no debe superar los 3 meses para decidir, ya sea por una exploración del nervio radial o el tratamiento definitivo de la parálisis. La ausencia de progresión de un signo de Tinel o la presencia de dolor neuropático son parámetros negativos por considerar en la interrupción del tratamiento conservador.

En pacientes con fracturas de alta energía donde la recuperación suele ser más lenta, suelo solicitar una ecografía para evaluar la continuidad nerviosa; si hay signos ecográficos de interrupción neural o atrapamiento nervioso, planifico la reconstrucción. Si la ecografía muestra la indemnidad, mantengo la conducta expectante, y acuerdo con el paciente el tiempo de expectación, que no sobrepasará los 6 meses.

\section{5. ¿Cuál es el tiempo que considera límite para realizar la exploración y la reconstrucción con injertos en una lesión del nervio radial a nivel del tercio medio del brazo? ¿Hay un tamaño del defecto límite?}

MS: A partir de los 6 meses desde la aparición de la parálisis, los resultados de una exploración y una eventual reparación suelen decaer rápidamente, por lo cual se recomienda hacerlo antes o en ese momento. ${ }^{13}$ Respecto a la longitud máxima de una reparación con injerto, tenemos mucho publicado sobre nuestra propia casuística de los resultados positivos obtenidos con injertos largos, inclusive de más de $20 \mathrm{~cm}$ de longitud, específicamente para transferencias nerviosas en el plexo braquial. El problema de usar injertos largos no radica en su extensión, sino en la extensión del daño primario que ha sufrido el nervio. En otras palabras, si necesitamos colocar un injerto de $15 \mathrm{~cm}$, por ejemplo, en un nervio radial, ello implica un daño equivalente al nervio afectado, un resultado pobre o nulo, y por ello que esto no se puede asemejar a las antes mencionadas transferencias con injertos largos en el plexo braquial. Para ser conciso luego de estas consideraciones, yo diría que si debemos utilizar injertos más largos de 10 a $12 \mathrm{~cm}$, deberíamos considerar abandonar la reparación primaria y plantearnos otras alternativas terapéuticas, como transferencias tendinosas o nerviosas, que tendrán un resultado positivo más predecible.

SV: Suelo explorar tempranamente las parálisis del nervio radial, y si detecto una severa lesión o sección suelo optar por la reconstrucción con injerto. Con excepción de las heridas cortantes, el nervio suele sufrir un traumatismo de compresión o de elongación que afecta un sector intraneural variable, y el éxito de una sutura nerviosa estará condicionado a que se la realice sin tensión, de un cabo proximal y distal sanos; por lo tanto, suelo usar injerto de nervio.

Los resultados con injertos suelen ser buenos, se recupera la extensión de la muñeca y, en menor medida, de los dedos, pero debe resaltarse que se han comunicado resultados pobres hasta en el $40 \%$ de los casos. Se obtienen mejores resultados en los defectos que requieren injertos de una longitud $<4 \mathrm{~cm}$ y se desaconseja la colocación de injerto en defectos de más de $10 \mathrm{~cm} \cdot{ }^{9,14}$

En pacientes con parálisis tardías (luego de los 3 meses), actualmente, prefiero realizar la reconstrucción de la función del nervio radial con transferencias nerviosas o tendinosas.

Los niños son una excepción, se puede esperar la recuperación espontánea hasta los 6 meses. Se logran resultados excelentes con injertos de nervio, incluso con injertos largos, probablemente debido al mayor potencial de regeneración y plasticidad neural. ${ }^{15}$ 


\section{6. ¿Qué factores son fundamentales a la hora de evaluar una lesión del nervio radial para considerar una reconstrucción primaria con injertos del nervio radial versus tratamiento con transferencias tendinosas?}

MS: La extensión de la lesión primaria, el tiempo transcurrido desde el trauma, la edad del paciente, el nivel de la lesión (distal o proximal, cuanto más distal mejor), entre otros, son todos factores que impactan en la reconstrucción primaria. La sumatoria de estos factores determinará la elección de una reparación con injertos o una transferencia, ya sea nerviosa o tendinosa.

SV: Son principalmente dos, los inherentes a la lesión y los del propio paciente.

En relación con la lesión, el tiempo transcurrido y el defecto neural son los más importantes. El tiempo guarda relación con el mínimo que llevará la reinervación y la degeneración propia de la placa motora, que se estima irreversible entre los 12 y 18 meses, y la degeneración muscular entre los 18 y 24 meses. ${ }^{9}$ No solo el tamaño de un injerto condiciona la recuperación, sino también el entorno donde se colocará, ya que un área con abundante fibrosis (heridas abiertas o infecciones) está poco vascularizada, y es menos favorable para la regeneración de los injertos. Las lesiones de nervio radial asociadas a lesiones vasculares muestran un menor potencial de recuperación.

Solo uso injertos en reconstrucciones tempranas, y no los recomiendo en una lesión de más de 6 meses. En reconstrucciones tardías, prefiero usar transferencias tendinosas o nerviosas.

Las comorbilidades y la edad del paciente son factores influyentes; después de los 50 años y en fumadores, los resultados suelen ser menos predecibles, y son candidatos a una transferencia tendinosa.

\section{7. ¿Cuál considera que sería la situación ideal para indicar transferencias nerviosas en un paciente con parálisis del nervio radial?}

MS: Se las indicaría a un paciente relativamente joven (hasta 50 años) en quien no se puede o no se pudo realizar una reparación con injertos, que no tenga problemas en esperar meses hasta obtener un resultado favorable y que jerarquice la obtención de una movilidad independiente en la extensión de cada dedo como resultado final. También el paciente tiene que asumir que si el resultado no es bueno se podría ver afectada parcialmente una eventual y ulterior transferencia tendinosa.

SV: Un paciente joven con una lesión de alta energía y condiciones locales que auguran un pronóstico malo de la reconstrucción del nervio, que no se recuperó pasados 4 meses de la lesión o si, durante la cirugía de exploración del nervio radial, se observa una lesión que necesita injerto con un defecto $>4 \mathrm{~cm}$.

\section{8. ¿Cuál es el esquema de transferencias nerviosas utilizado en su práctica diaria en parálisis altas y bajas del nervio radial? Fundamente brevemente su elección.}

MS: En las lesiones bajas, una rama del flexor superficial de los dedos al extensor corto del carpo y una rama del palmar mayor al nervio interóseo posterior. En las lesiones altas, sumaría a los dadores axonales anteriores provenientes del mediano uno del cubital, específicamente ramas del cubital anterior -como en la cirugía de Oberlin-, pero para el nervio de la porción larga del tríceps. ${ }^{16}$ Estas técnicas son las más probadas y las más seguras (menor riesgo funcional generado para el nervio dador).

SV: Prefiero las transferencias "híbridas". Uso la transferencia tendinosa del pronador redondo al extensor radial del carpo corto, porque, según mi experiencia, otorga resultado más rápidos y predecibles.

En pacientes jóvenes, motivados, no fumadores, he realizado la reinervación del nervio interóseo posterior, usando fascículos del flexor radial del carpo, con resultados no tan buenos como los publicados. Después de 2017, cuando el doctor Rui Ferreira (Recife, Brasil) me enseñó su técnica, y pude evaluar sus resultados en misiones humanitarias, prefiero transferir la rama terminal del nervio interóseo anterior, que inerva al pronador cuadrado, a la rama anterior del nervio interóseo posterior que inerva al extensor común de los dedos, el extensor propio del meñique y el extensor cubital del carpo, asociado a una transferencia tendinosa del pronador redondo al extensor corto radial del carpo y a un "re-routing" del palmar largo al extensor largo del pulgar. Esta técnica (aún no publicada) consigue una buena extensión de los dedos conservando la individualidad, sin perder potencia en los flexores de la muñeca y los dedos. En parálisis bajas, exploro el nervio interóseo posterior y, si es posible, hago la misma transferencia nerviosa y un "re-routing" del palmar largo al extensor largo del pulgar. 


\section{9. ¿Cuáles son los beneficios, según la bibliografía y su práctica diaria, por los cuales inclinaría su elec- ción a realizar transferencias nerviosas por sobre las tendinosas?}

MS: Las transferencias nerviosas tienen, a mi parecer, dos desventajas: 1) sus resultados, si bien de acuerdo con lo publicado son buenos, ${ }^{17,18}$ las series y el número de pacientes son escasos, y tienden a provenir de un grupo no tan grande de autores y 2) los resultados tardan de 8 a 12 meses y requieren una intensiva rehabilitación. En contraposición, las transferencias tendinosas para la parálisis radial son procedimientos versátiles y confiables con resultados positivos a lo largo de décadas de experiencia con innumerables reportes y experiencias personales, son enteramente reproducibles y no requieren un entrenamiento especial en microcirugía nerviosa. Se podría decir que la movilidad independiente para la extensión individual de cada dedo no es tan buena con las transferencias tendinosas. Por lo antedicho y aunque suene extraño proviniendo de un neurocirujano dedicado a cirugía de nervios, me inclino actualmente por las transferencias tendinosas en las parálisis radiales, tanto cuando haya fracasado una reconstrucción primaria como ante la imposibilidad o demora en realizarla. Por supuesto, esta conducta podría cambiar en el futuro si las transferencias nerviosas, que han sido descritas mucho más recientemente que las tendinosas, demuestran una confiabilidad similar a la de estas últimas.

SV: Considero que las transferencias nerviosas se muestran como una prometedora opción, pero aún no existe suficiente evidencia que demuestre que sus resultados sean superiores a los de las clásicas transferencias tendinosas en las parálisis del nervio radial. ${ }^{19}$ En mi opinión, su principal ventaja es que consiguen individualidad de la extensión de los dedos, algo que no se obtiene con las transferencias tendinosas, fuera de la individualidad de extensión obtenida de la interfalángica proximal e interfalángica distal a expensas de los intrínsecos. En contraparte, el tiempo de recuperación es más prolongado y existe el riesgo de una recuperación incompleta. ${ }^{19}$ Bertelli comparó las transferencias nerviosas con las tendinosas y observó similares resultados, pero a favor de las transferencias nerviosas, una mayor fuerza de puño, sin la tendencia a la desviación lateral o la pérdida de algunos grados de flexión de la muñeca, y sin hiperextensión compensadora de la metacarpofalángica del pulgar a la extensión, inclinándose claramente en favor de las transferencias nerviosas. ${ }^{20}$

Las transferencias tendinosas son seguras, tienen resultados previsibles, que permiten una recuperación más rápida, con opciones adaptables a cada paciente y la posibilidad de revisión, algo difícil de hacer en una transferencia nerviosa. Su limitación es la falta de individualidad de la extensión de los dedos, esto justifica mi tendencia a los procedimientos "híbridos".

\section{0. ¿Cuál es su técnica de primera elección para el manejo de un neuroma de rama superficial sensitiva del nervio radial? Y, en caso de fracaso, ¿cuál es la alternativa contemplada para la resolución?}

MS: La primera elección es la reconstrucción nerviosa, directa de preferencia o con injertos. La gran molestia y la incapacidad funcional que generan este tipo de lesiones justifican la morbilidad que causa la toma del injerto, generalmente del nervio sural o safeno externo, pero si el defecto nervioso es $<3 \mathrm{~cm}$ se podrán usar tubos como para los neuromas digitales. La reconstrucción impide o disminuye la formación de un neuroma proximal, que es la fuente de molestias. En segunda instancia, se puede seccionar el nervio más proximalmente de donde está lesionado, y colocarlo profundamente en músculo o tejido laxo. Esta variante no suele ser tan eficaz como la anterior. Otra alternativa para evitar la formación de un neuroma, originalmente descrita para los neuromas en amputados es la llamada Targeted Muscle Reinnervation, ${ }^{21}$ que consiste en unir al nervio radial superficial con alguna rama motora sacrificable. El tratamiento del neuroma doloroso de cualquier localización es un problema de difícil solución, de ahí la gran cantidad de métodos descritos para tratarlo y la inexistencia de uno que se imponga sobre el resto.

SV: Realizo la resección del neuroma, la ligadura del nervio y su transposición intramuscular, sin tensión en profundidad. Según la localización, profundizo en el braquirradial o en el pronador cuadrado. En recidivas, si la longitud del nervio lo permite, llevo a cabo una relocalización intraósea en el radio; si la distancia del nervio no lo admite, opto por una sutura microquirúrgica termino-lateral sobre sí mismo ("nerve loop”) y su profundización más proximal.

He abandonado los intentos de reconstruir la sensibilidad con injertos de nervios o neurotubos y técnicas de envoltura ("vein wrap") en el nervio radial, ya que su superficialidad no permite que desaparezcan completamente los síntomas y la pérdida de la sensibilidad en su territorio es bien tolerada. 
Conflicto de intereses: Los autores no declaran conflictos de intereses.

\section{BIBLIOGRAFÍA}

1. Shao YC, Harwood P, Grotz MR, Limb D, Giannoudis PV. Radial nerve palsy associated with fractures of the shaft of the humerus: a systematic review. J Bone Joint Surg Br 2005;87(12):1647-52. https://doi.org/10.1302/0301-620X.87B12.16132

2. Bumbasirevic M, Palibrk T, Lesic A, Atkinson H. Radial nerve palsy. EFORT Open Rev 2017;1(8):286-94. https://doi.org/10.1302/2058-5241.1.000028

3. Chang G, Ilyas AM. Radial nerve palsy after humeral shaft fractures: The case for early exploration and a new classification to guide treatment and prognosis. Hand Clin 2018;34(1):105-12. https://doi.org/10.1016/j.hcl.2017.09.001

4. Hegeman EM, Polmear M, Scanaliato JP, Nesti L, Dunn JC. Incidence and management of radial nerve palsies in humeral shaft fractures: a systematic review. Cureus 2020;12(11): e11490. https://doi.org/10.7759/cureus.11490

5. Ilyas AM, Mangan JJ, Graham J. Radial nerve palsy recovery with fractures of the humerus. J Am Acad Orthop Surg 2020;28(6):e263-e269. https://doi.org/10.5435/JAAOS-D-18-00142

6. Shah A, Jebson PJ. Current treatment of radial nerve palsy following fracture of the humeral shaft. J Hand Surg Am 2008;33(8):1433-4. https://doi.org/10.1016/j.jhsa.2008.05.029

7. Ring D, Chin K, Jupiter JB. Radial nerve palsy associated with high-energy humeral shaft fractures. J Hand Surg Am 2004;29(1):144-7. https://doi.org/10.1016/j.jhsa.2003.09.013

8. Venouziou AI, Dailiana ZH, Varitimidis SE, Hantes ME, Gougoulias NE, Malizos KN. Radial nerve palsy associated with humeral shaft fracture. Is the energy of trauma a prognostic factor? Injury 2011;42(11);1289-93. https://doi.org/10.1016/j.injury.2011.01.020

9. Laulan J. High radial nerve palsy. Hand Surg Rehabil 2019;38(1):2-13. https://doi.org/10.1016/j.hansur.2018.10.243

10. Fu SY, Gordon T. Contributing factors to poor functional recovery after delayed nerve repair: prolonged denervation. J Neurosci 1995;15(5 Pt 2):3886-95. https://doi.org/10.1523/JNEUROSCI.15-05-03886.1995

11. Shaw JL, Sakellarides H. Radial-nerve paralysis is associated with fractures of the humerus. A review of forty-five cases. J Bone Joint Surg Am 1967;49(5):899-902. PMID: 6029259

12. Amillo S, Barrios RH, Martínez-Peric R, Losada JI. Surgical treatment of the radial nerve lesions associated with fractures of the humerus. J Orthop Trauma 1993;7(3):211-5. https://doi.org/10.1097/00005131-199306000-00002

13. Kline DG. Nerve surgery: where we are and where we might go. Neurosurg Clin North Am 2008;19(4):509-16, v. https://doi.org/10.1016/j.nec.2008.07.006

14. Shergill G, Bonney G, Munshi P, Birch R. The radial and posterior interosseous nerves. Results of 260 repairs. $J$ Bone Joint Surg Br 2001;83(5):646-9. https://doi.org/10.1302/0301-620x.83b5.11312

15. Bertelli J, Soldado F, Ghizoni MF. Outcomes of radial nerve grafting in children after distal humerus fracture. $J$ Hand Surg Am 2018;43(12):1140.e1-1140.e6. https://doi.org/10.1016/j.jhsa.2018.04.018

16. Emamhadi M, Andalib S. The first experience of triple nerve transfer in proximal radial nerve palsy. World Neurosurg 2018;109:351-5. https://doi.org/10.1016/j.wneu.2017.10.033

17. Mackinnon SE, Donor distal, recipient Proximal and other personal perspectives on nerve transfers. Hand Clin 2016;32:141-51. https://doi.org/10.1016/j.hcl.2015.12.003

18. Moore AM, Franco M, Tung TH. Motor and sensory nerve transfers in the forearm and hand. Plast Reconstr Surg 2014;134(4):721-30. https://doi.org/10.1097/PRS.0000000000000509

19. Compton J, Owens J, Day M, Caldwell L. Systematic review of tendon transfer versus nerve transfer for the restoration of wrist extension in isolated traumatic radial nerve palsy. J Am Acad Orthop Surg Glob Res Rev 2018;2(4):e001. https://doi.org/10.5435/JAAOSGlobal-D-18-00001

20. Bertelli JA. Nerve versus tendon transfer for radial nerve paralysis reconstruction. J Hand Surg Am 2020;45(5):41826. https://doi.org/10.1016/j.jhsa.2019.12.009

21.Dumanian GA, Potter BK, Mioton LM, Ko JH, Cheesborough JE, Souza JM, et al. Targeted muscle reinnervation treats neuroma and phantom pain in major limb amputees: a randomized clinical trial. Ann Surg 2019;270(2):23846. https://doi.org/10.1097/SLA.0000000000003088 